\title{
Liderazgo e inteligencia emocional
}

\section{Leadership and emotional intelligence}

Santiago Nájera, Ph.D. Candidate

Universidad Central del Ecuador, Ecuador

Autor para correspondencia: snajera@pucp.pe

Fecha de recepción: 05 de Enero de 2016 - Fecha de aceptación: 27 de Enero de 2016

\section{Resumen}

El Liderazgo e inteligencia emocional juega un papel central entre las interacciones de los actores y observadores de las organizaciones. No es sino en los últimos años que se ha despertado el creciente interés en el estudio de la inteligencia emocional por parte de las empresas. Este estudio presentará que la clave para el éxito en liderazgo son resultado de interacciones sociales y no únicamente temas de desempeño operativo, de aquí que se puede suponer que aquellos líderes con mayor desarrollo habilidades sociales y por ende mayor grado de inteligencia emocional podrán conducir de mejor manera a sus seguidores y a la organización a alcanzar sus objetivos

Palabras claves: liderazgo; inteligencia emocional; empresas; éxito empresarial

\begin{abstract}
Leadership and emotional intelligence plays a central role interactions between actors and observers of organizations. It is only in recent years that has attracted growing interest in the study of emotional intelligence on the part of companies. This study will present the key to successful leadership are the result of social interactions and not just issues of operational performance, hence it can be assumed that those leaders most developed social skills and thus greater emotional intelligence can lead to better so his followers and the organization achieve its objectives
\end{abstract}

Key words: leadership; emotional intelligence; business; business success 


\section{Introducción}

El liderazgo sin lugar a duda es un proceso de interacción social donde los líderes buscan influir sobre sus seguidores (Yukl, Gordon, \& Taber, 2002), de aquí que se puede definir el liderazgo dentro de los términos de la psicología social, donde la inteligencia emocional juega un papel central entre las interacciones de los actores y observadores de las organizaciones. Los líderes pueden ser guiados por reglas y procedimientos, pero deben interiorizar en ellos la misión de lo que buscan hacer para poder de esta manera a los otros su visión y lograr de esta manera inspirarlos a un grupo a que se transformen en sus seguidores.

No es sino en los últimos años que se ha despertado el creciente interés en el estudio de la inteligencia emocional por parte de las empresas (Fineman, 1997), se encuentra en el constante interés de mantener ventaja competitiva por parte de las organizaciones, pese a esto pocos estudios se han realizado al respecto desde el punto de vista de las organizaciones, y más bien han sido centrados desde el desarrollo psicológico. El tema de inteligencia emocional ha sido sin lugar a duda impulsado por el libro de Daniel Goleman (Daniel, 1996), quien define a este constructo en tres partes: (a) entender que siente uno y ser capaz de manejar esos sentimientos, (b) tener la habilidad de auto-motivarse en lograr objetivos, y (c) entender lo que otros sienten y manejar esta relaciones de manera efectiva; una definición más clara de inteligencia emocional es la que plantea Martínez (1997) al decir que es un grupo de habilidades no cognitivas, capacidades y competencias que influencia las habilidades personales para cumplir con las necesidades y presiones del medio.

La necesidad del estudio de la inteligencia emocional tiene su origen en la imposibilidad del uso de la medición tradicional de la inteligencia racional para determinar el suceso de las personas en la vida, es así que estudios han demostrado que los líderes tienden a ser más inteligentes que el promedio del grupo, pero no los más inteligentes (Bahn, 1979), de aquí que se han realizado múltiples estudios relacionados a la inteligencia siendo Gardner y Hatch (1989) quienes desarrollaron el concepto de inteligencias múltiple u otras inteligencias, analizando la inteligencia desde un constructo diferente al del coeficiente intelectual.

\section{Impacto y beneficio de la inteligencia emocional}

Un punto de vista popular sobre la inteligencia emocional da a esta más importancia que al coeficiente intelectual, no obstante el mismo Goleman (1996) plantea que ambas no son opuestas entre sí, pero se encuentran separadas la una de la otra, por lo cual la combinación de ambas produce un aprendizaje exitoso al igual que un desempeño exitoso, es así que la combinación de ambas es más importante que únicamente el coeficiente intelectual, adicionalmente se ha visto que la inteligencia emocional tiene un mayor impacto en el logro de objetivos, de aquí que autores como Harrison (1997) manifiestan que la inteligencia emocional están en el centro del desarrollo de las ventajas competitivas de las organizaciones.

En relación al proceso del aprendizaje de la gestión, Fineman (1997) desarrolla la idea de que existe una relación directa entre emoción y competencias, puesto lo que se conoce como supra-competencias incluye competencias interpersonales entre las cuales asertividad, persuasión, decisión, sensibilidad y comunicación oral. 
Teniendo en cuenta la significancia de la inteligencia emocional como factor diferenciado en términos del éxito personal y por tanto del éxito de las organizaciones, resalta la pregunta si es posible o no medir la inteligencia emocional, desde el uso de avanzadas pruebas como el MSCEIT V2.0 (Mayer, Salovey, Caruso, \& Sitarenios, 2003) hasta pruebas con papel y lápiz, siendo los comentarios al respecto muy polarizados, incluso el mismo Goleman (1996) señala que no existe al momento ninguna prueba que pueda calificar o medir la inteligencia emocional y que probablemente nunca existirá, de aquí que autores como Martínez (1997) señalan que probablemente la retroalimentación de jefes y subordinados se la manera más apropiada de medir este constructo, antes que el uso de una prueba con papel y lápiz.

Al igual que la dificultad en medir la inteligencia emocional, otro tema difícil de medir es el éxito en la vida y en las organizaciones, es así que autores como Dulewicz y Herbert (1996; 1999) plantean que el éxito en las organizaciones puede ser medido como el progreso dentro del orden jerárquico de la organización, para lo cual plantean el comprar el nivel jerárquico, remuneración, número de personas que le reportan, etc.

La relación positiva entre la inteligencia emocional y el liderazgo transaccional ha sido revisada ya por múltiples autores (Ashkanasy \& Tse, 2000; Friedman, Riggio, \& Casella, 1988; George, 2000), los cuales indican que líderes con alto desarrollo de inteligencia emocional, son mejores en el manejo de las emociones de sus empleados, haciendo que estos se comporten de manera eficiente en la consecución de objetivos y tengan un mayor nivel de satisfacción por el trabajo que realiza, no obstante otros autores (Ashkanasy \& Daus, 2002; Dasborough \& Ashkanasy, 2002) señalan que el alto desarrollo de la inteligencia emocional puede ser utilizado también para manipular a los trabajadores con el objetivo de utilizarlos para el logro de objetivos personales por parte del líder, haciendo que sus seguidores simplemente se conviertan en un recurso.

Es importante recordar que los líderes transformacionales inspiran a sus seguidores a una visión y objetivos claros, de manera que los miembros de la organización se sientan motivados y fuertemente conectada a la líder, esta motivación se produce cuando el líder ha mostrado confianza y entusiasmo, y además ha logrado contar con un conocimiento de las necesidades emocionales de los miembros del equipo (Cherulnik, Donley, Wiewel, \& Miller, 2001), por tanto, según lo sugerido por George(2000), el liderazgo transformacional es aquel que se basa fundamentalmente en los procesos de relaciones personales y emocionales.

Es así que Weierter (1997) argumenta que los miembros de una organización reaccionan de manera particular a la personalidad carismática del líder o a los mensajes carismáticos del líder, pero que la interpretación de este tipo de liderazgo depende a la vez de los atributos personales de cada uno de los miembros del grupo, adicionalmente Conger (1988) señala que un líder carismático puede tener un impacto inusual en algunos miembros del equipo, haciendo que estos tengan una voluntad incuestionable a obedecer instrucciones de su líder, adicionalmente el mismo autor que la capacidad que el líder tiene la capacidad de gestionar las emociones de sus seguidores y logra crear y comunicar una visión sobre la organización, no obstante estas habilidades se pueden usar para el bien así como para el mal, lo que puede conducir a comportamientos destructivos, tales como afirmaciones exageradas sobre la visión, la 
manipulación de las audiencias y la distracción de los seguidores sobre los resultados negativos del líder o de la organización, es así que Owen (1986) señala que el líder tiene la habilidad para manipular y gestionar a los recurso humanos.

Desde una visión positiva Ferris, et al.,(1995) sostienen que una manipulación por parte de un actor es el resultado de la interacción entre el perceptor y la percepción, es así que los verdaderos líderes transformacionales logran transformar su organización a través de seguidores que tienden a unirse a ellos en la consecución de sus metas visionarias que busque el bien común de todos los miembros de la organización y no solamente alcanzar los objetivos personales del lider. Bass y Steidlmeier (1999) también señalan que un liderazgo transformacional contiene una sólida base ética y moral, de aquí que el verdadero liderazgo transformacional es una forma positiva de liderazgo.

Las personas tienden a analizar y entender la conducta de otras personas a través de la percepción delas intenciones del otro (Thomas \& Pondy, 1977), no obstante el comportamiento real sólo puede llegar a ser interpretado si la intención que subyace a la acción que se identificó al inicio (Fishbein \& Ajzen, 1975), de aquí la importancia de que los seguidores perciban las intenciones del líder como acciones positivas en pro del desarrollo personal del seguidor, caso contrario este podrá sentir que está siendo utilizado por el líder, de aquí ya hace la importancia que las acciones realizadas por el líder tenga verdaderamente como fin el bienestar del grupo y no únicamente el de este.

Adicionalmente George (2000) considera que el estado de ánimo del puede influir tanto positiva como negativamente en el desempeño del grupo, encontrando que ante el estado de ánimo positivo, los miembros de la organización son más propensos a atribuir la conducta del líder como sincera y bien intencionada, mientras que ante estados de ánimo negativos los miembros del grupo asocian la conducta del líder como egoísta y manipuladora.

Calder (1977) señala que el liderazgo es un proceso de interacción social, de aquí que los estados de ánimo del líder influyen en cómo los miembros perciben la conducta del líder, por tanto se puede proponer que la inteligencia emocional es una característica importante de un líder, ya que el papel de este consiste en percibir y entender las emociones propias y las emoción que evoca en otros (Ashforth \& Humphrey, 1995; Ashkanasy \& Tse, 2000), con el fin de poder influenciar sobre los otros, es así que los verdaderos líderes transformacionales y pseudo transformacional exhiben conductas similares, que tienden a mejorar a través del grado de desarrollo de la inteligencia emocional (George, 2000) por tanto aquellos líderes con alta desarrollo de la inteligencia emocional tienen mayor control de sus emociones y son más propensos a presentar una impresión de que se su comportamiento es transformación, independientemente de sus intenciones o motivos.

Es así que autores como Arlie Hochschield (1979) plantearon los conceptos de emociones organizacionales y emociones del trabajo, conceptos que posteriormente seria la base para las teorías de comportamiento organizacional, es así que con estos conceptos se explica las ideas de que existen emociones interrelacionadas entre los miembros de la organización, y que es el líder quien intrínsecamente reorganiza los estados anímicos de los trabajadores, y después busca como manejar los estados anímicos de los trabajadores de manera coordinada (Ashforth \& Humphrey, 
1993), por tanto podemos ver que las emociones juegan un rol importante en el conocimiento de las organizaciones, y por tanto tienen un papel importante en la enseñanza de la gestión y del liderazgo, puesto se requiere del entendimiento y el análisis de la inteligencia emocional de los trabajadores con el objetivo de lograr una gestión estrategia efectiva (Ashkanasy, Härtel, \& Zerbe, 2000).

\section{Conclusiones}

Cada vez el estudio de la inteligencia emocional en el éxito de las personas y las empresas toma mayor importancia, principalmente debido a que el manejo de una organización y el liderazgo son resultado de interacciones sociales y no únicamente temas de desempeño operativo, de aquí que se puede suponer que aquellos líderes con mayor desarrollo habilidades sociales y por ende mayor grado de inteligencia emocional podrán conducir de mejor manera a sus seguidores y a la organización a alcanzar sus objetivos (Owen, 1986), no obstante también queda claro que dichas habilidades utilizados por falsos líderes transformacionales pueden ser utilizadas de manera destructiva para el grupo y organización en beneficio único del líder. Por tanto la importancia de comprender que es la inteligencia emocional y como esta se ve relacionada con las característica de un líder, y como el correcto uso de estas características puede generar una diferencia competitiva en las organizaciones de manera tal que las vuelva más competitivas y por tanto puedan alcanzar su objetivos tanto en el mediano y largo plazo.

Si bien autores como Goleman (1996) han planteado que la inteligencia emocional es la diferencia entre el éxito o el fracaso de una personas (Victor Dulewicz \& Herbert, 1999), existe múltiples críticas al respecto de que si es únicamente este tipo de inteligencia la que puede brindar a una persona una ventaja sobre el resto, lo que si se encuentra claro es que un requerimiento que el líder cuente con un buen grado de desarrollo de su inteligencia emocional para poder entender sus emociones y las del resto de sus seguidores (Ashkanasy \& Daus, 2002), para de esta manera poder guiarlos de manera adecuada.

\section{Bibliografía}

Ashforth, B. E., \& Humphrey, R. H. (1993). Emotional labor in service roles: The influence of identity. Academy of Management Review, 18(1), 88-115.

Ashforth, B. E., \& Humphrey, R. H. (1995). Emotion in the workplace: A reappraisal. Human relations, 48(2), 97-125.

Ashkanasy, N. M., \& Daus, C. S. (2002). Emotion in the workplace: The new challenge for managers. The Academy of Management Executive, 16(1), 76-86.

Ashkanasy, N. M., Härtel, C. E., \& Zerbe, W. J. (2000). Emotions in the workplace: Research, theory, and practice: Greenwood Publishing Group.

Ashkanasy, N. M., \& Tse, B. (2000). Transformational leadership as management of emotion: A conceptual review.

Bahn, C. (1979). Can Intelligence-Tests Predict Executive Competence. Personnel, 56(4), 52-58.

Bass, B. M., \& Steidlmeier, P. (1999). Ethics, character, and authentic transformational leadership behavior. The Leadership Quarterly, 10(2), 181-217.

Calder, B. J. (1977). An attribution theory of leadership. New directions in organizational behavior, 179, 204. 
Cherulnik, P. D., Donley, K. A., Wiewel, T. S. R., \& Miller, S. R. (2001). Charisma Is Contagious: The Effect of Leaders' Charisma on Observers' Affect1. Journal of Applied Social Psychology, 31(10), 2149-2159.

Conger, J. A., \& Kanungo, R. N. (1988). Charismatic leadership: The elusive factor in organizational effectiveness: Jossey-Bass.

Daniel, G. (1996). La inteligencia emocional. Kairós. Barcelona.

Dasborough, M. T., \& Ashkanasy, N. M. (2002). Emotion and attribution of intentionality in leader-member relationships. The Leadership Quarterly, 13(5), 615-634.

Dulewicz, V., \& Herbert, P. (1996). General Management Competences and Personality: A 7 Year Follow-up Study. Working Paper Series-Henley Management College Hwp.

Dulewicz, V., \& Herbert, P. (1999). Predicting Advancement to Senior Management from Competencies and Personality Data: A Seven-year Follow-up Study. British Journal of Management, 10(1), 13-22.

Ferris, G., Bhawuk, D., Fedor, D., \& Judge, T. (1995). Organizational politics and citizenship: Attributions of intentionality and construct definition. Advances in attribution theory: An organizational perspective, 231-252.

Fineman, S. (1997). Emotion and management learning. Management Learning, 28(1), 13-25.

Fishbein, M., \& Ajzen, I. (1975). Belief, attitude, intention and behavior: An introduction to theory and research.

Friedman, H. S., Riggio, R. E., \& Casella, D. F. (1988). Nonverbal skill, personal charisma, and initial attraction. Personality and Social Psychology Bulletin, 14(1), 203-211.

Gardner, H., \& Hatch, T. (1989). Educational implications of the theory of multiple intelligences. Educational researcher, 18(8), 4-10.

George, J. M. (2000). Emotions and leadership: The role of emotional intelligence. Human relations, 53(8), 1027-1055.

Harrison, R. (1997). Why your firm needs emotional intelligence. People Management, 3(1), 41.

Hochschild, A. R. (1979). Emotion work, feeling rules, and social structure. American journal of sociology, 551-575.

Martinez, M. N. (1997). The smarts that count. HR magazine, 42(11), 72-78.

Mayer, J. D., Salovey, P., Caruso, D. R., \& Sitarenios, G. (2003). Measuring emotional intelligence with the MSCEIT V2. 0. Emotion, 3(1), 97.

Owen, H. (1986). Leadership indirection. Transformational leadership, 111-122.

Thomas, K. W., \& Pondy, L. R. (1977). Toward an" intent" model of conflict management among principal parties. Human relations, 30(12), 1089-1102.

Weierter, S. J. (1997). Who Wants to Play "Follow the Leader?" A theory of charismatic relationships based on routinized charisma and follower characteristics. The Leadership Quarterly, 8(2), 171-193.

Yukl, G., Gordon, A., \& Taber, T. (2002). A hierarchical taxonomy of leadership behavior: Integrating a half century of behavior research. Journal of Leadership \& Organizational Studies, 9(1), 15-32. 